\title{
Robust Stability of Networked Control Systems with Communication Constraints
}

\author{
Yasir B. Hamdan and Samah O. Altayeb
}

\begin{abstract}
This paper investigates a robust state feedback controller for networked control systems (NCSs) with a finite bandwidth and network congestion. A simple scheme is used in order to eliminate the effect of the congestion, considering the existence of arbitrary packet dropout. A mathematical model of NCS with uncertain, varying and bounded network induced delay is obtained. The model is then converted into uncertain switching system model. The stability criteria for closed-loop system are formulated and sufficient conditions for the controller are given in terms of solvability of linear matrix inequalities (LMIs). An illustrative example is used to evaluate how effective the simple congestion control scheme is in reducing network bandwidth and preserving the stability of the NCS.
\end{abstract}

Index Terms-Networked control system, network congestion, linear matrix inequalities.

\section{INTRODUCTION}

Networked control systems (NCSs) are feedback control systems with network channels used for the communications between spatially distributed system components, such as sensors, actuators, and controllers. NCSs received increasing interests in recent years for the advantages of low cost, reduced weight and power requirements, flexibility and ease of maintenance. However, the insertion of communication networks introduce some new problems such as network-induced delay, data packet dropout and access constraints, which can degrade control performance and even lead to instability. Since conventional control methods do not take these problems introduced by communication network into account, the study of new control strategies for NCS is of practical importance. A rapid development of analysis and synthesis of NCSs, and new research results have continually been reported in literatures, such as [1]-[6].

In order to achieve stability requirements, controller synthesis methodologies have been proposed using Lyapunov functions stability analysis [3], [7] and control synthesis [8]. The NCSs stability analysis and controller design with long time delay presented in [9]. And [10], proposed a combined delay switching and parameter uncertainty-based method to deal with time-varying delay and Packet dropout. Paper [11], study the stability of the NCSs under long delay and packets reordering assumption. A buffer is located at the receiving end of the channels for randomly delayed packets, which releases them at regular intervals [12].

Manuscript received April 5, 2014; revised June 30, 2014.

Y. B. Hamdan and S. O. Altayeb are with the Department of Automation, University of Science and Technology of China, Hefei 230026, China (e-mail: yasir@mail.ustc.edu.cn).
Most of the above mentioned communication constraints, are imposed by either of the facts that the communication medium in NCS is shared by node and that it has limited data rate or insufficient band width i.e., the limitation of capacity or throughput. Very few studies include the time triggered sampling approach considers the plant dynamics and communication constraint, such as finite bandwidth and network congestion. In NCSs congestion can be defined as, the controllers receives the packets faster than they forward them. One of two things must be happen i) the subsystem prevent additional packets from entering congested region until those already present can be processed ii) the congested controller can discard queued packet to make rooms for those that are arriving.

Generally, in NCS data transfer treated with low level protocol such as Ethernet and TDMA. The challenge on the NCS, congestion control involves the optimization of performance in face of constraints on communication band width and communication resource to meet the soft and hard real time requirements [13], [14]. For example the different type of data exist in an NCS, hence different protocol or different priorities for packet can be used, a comparison among several congestion control schemes used by current transport protocol for NCSs is presented [15].

The NCS block diagram shown in Fig. 1 present network induced delays $\tau_{k}^{s c}$ is the communication delay between the sensor and the controller, $\tau_{k}^{c}$ is the computational delay in the controller and $\tau_{k}^{c a}$ is the communication delay between the controller and the actuator.

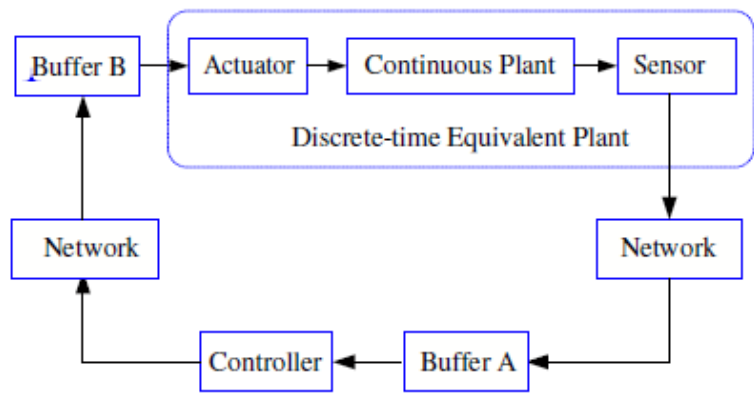

Fig. 1. The structure of concerned networked control system.

We assume that the sensor acts in a time-driven fashion and has identical sampling period $h$, and that both the controller and the actuator act in an event-driven fashion (i.e. calculation of the new control or actuator signal as soon as newly data arrived). For the time invariant controllers, all three delays can be captured by a single delay $0<\tau_{k}=$ $\tau_{k}^{s c}+\tau_{k}^{c}+\tau_{k}^{c a} \leq \bar{d} h$, i.e., the delay $\tau_{k}$ is uncertain can be evaluated between two adjacent sampling period, namely, $\tau_{k} \in\left[0, \tau_{\max }\right]$ with $\tau_{\max } \in[0, \bar{d} h]$ and $\bar{d}=$ $\left\lceil\tau_{\text {max }} / h\right\rceil$ where , $\lceil\diamond\rceil$ is the least integer larger than $\diamond$. In this 
work we focus on eliminating the effect of the congestion, we assume a queue buffer is maintained at the receiving end of the channels works in DropTail (Queue based congestion control scheme simply drops the incoming subsequent packets when the queue becomes full.) manner [15]. Hence we consider a deterministic packet dropout and the number of successive subsequent are bounded and the bound is denoted by $\bar{\delta}$, where $\bar{\delta} \in \mathbb{N}$. Furthermore, as the consequence of large delay, the out of order data will be rejected.

In this paper, we focus on solving the state feedback controller design problem of NCSs in discrete-time domain and under a general framework, where large network induced delay and arbitrary packet dropout are taken into account simultaneously. The mathematical model is proposed using similar technique in [8], [16]. It proposes a switched delay-based method to model the NCSs, and then the combined delay switching and parameter uncertainty based method is proposed. In terms of the given model, we give sufficient conditions for the existence of state feedback controller such that the closed-loop NCSs are robust asymptotically stable. Based on the obtained stability conditions, we further investigate the corresponding state feedback controller parameter uncertainty based method. Numerical examples are provided to demonstrate the effectiveness of the proposed approaches.

Section II deal with the modeling aspects of NCS with both delay and packet drop as switched system. In Section III, the NCS problem formulation is presented focusing on the issues of robust stability analysis and control synthesis. Numerical example follows in Section IV, while conclusion appears in the last section.

Notation: Throughout this article, $M^{T}$ represents the transpose of matrix $M$. I and $O$ represent identity matrices and zero matrices with appropriate dimensions, respectively. $\sigma_{\text {max }}(M)$ is the maximum singular value of matrix $M$. furthermore, $x_{k}$ will refer to the value $x(k h)$ of discrete-time signal of periodic samples.

\section{PROBLEM DESCRIPTIONS}

The structure of the considered NCS with network induced delay and packet dropout is shown in Fig.1, where the plant description is described by the following continuous-time linear system model

$$
\begin{aligned}
& x(t)=A x(t)+B u^{*}(t) \\
& y(t)=C x(t), u^{*}(t)=u_{k(t)}
\end{aligned}
$$

where, $x(t) \in \mathbb{R}^{n}$ state of the plant, $u^{*}(t) \in \mathbb{R}^{m}$ the most recently received control variable is assumed to remain active in the plant since no new updates i.e., packet dropped out. $y(t) \in \mathbb{R}^{p}$ the (measured) output of the plant, and $A, B$ and $C$ are known real constant matrices of appropriate dimension.

An example of the timing diagram of the considered NCS with both time delay and packet dropout, is shown in Fig. 2. in which the two controls signal $u_{k+1}$ and $u_{k+2}$ shown in dashed line are lost during $\left[s_{k+1}, s_{k+2}\right]$ and $\left[s_{k+2}, s_{k+3}\right]$ respectively. And the data rejected during the interval $\left[s_{k+7}, s_{k+8}\right]$. It can be seen from the timing diagram that the control input acting in the plant are different from one sampling interval to another sampling intervals, and thus the system model of the NCS varying from one sampling to another as the packet dropped out or rejected. To show that let $\tau_{k} \in[0,2 h], \forall k$, then the NCS model can be described into three main categories as follows:

1) There is no congestion; hence no packet will be dropped. A general case in which the number of active control inputs in one sampling interval is variable and depend on the current and previous time delay with $0 \leq \tau_{\min } \leq$ $\tau_{\text {max }} \leq \bar{d} h$, such situation in $\left[s_{k}, s_{k+1}\right],\left[s_{k+5}, s_{k+6}\right]$, and $\left[s_{k+7}, s_{k+8}\right]$. The last subsystem describes the case where data rejection occurs. Such NCSs modeling approach that consider different subsystems in [17], and include data rejection in [8].

2) The controller is congested, there are $i$ packet could be dropped out, and $0<i<\bar{\delta}$ such situation on the $\left[s_{k+1}, s_{k+2}\right]$ and $\left[s_{k+2}, s_{k+3}\right]$ in Fig. 2. The typical NCS mode is in [10], [18].

3) The controller is not congested, but there are $i$ successive dropout within the last sampling intervals, and $0<i<\bar{\delta}$. This case can be modeled same as in (1).

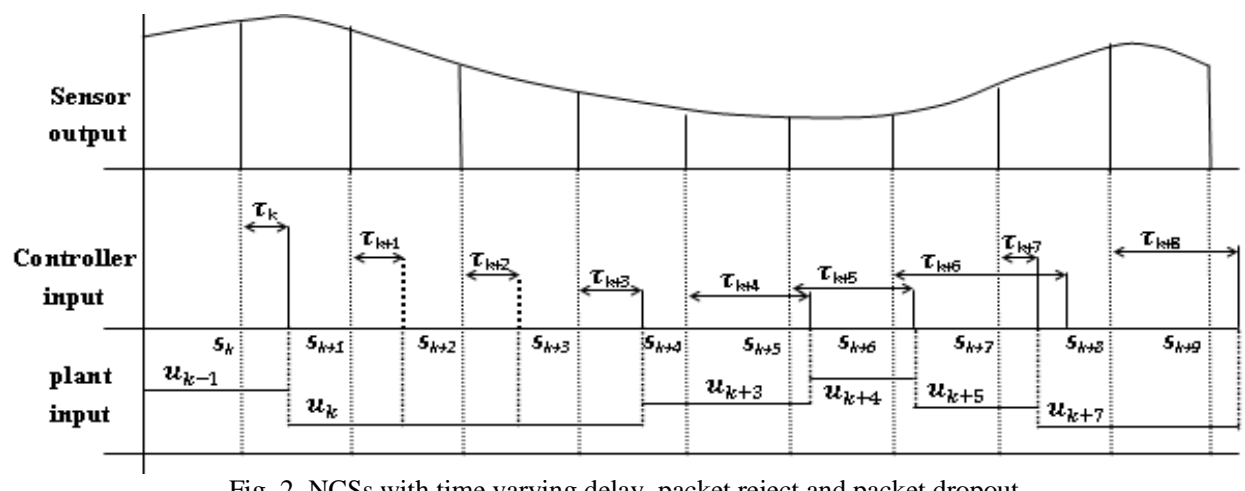

Fig. 2. NCSs with time varying delay, packet reject and packet dropout.

Remark 1: Obviously, the definition of $k(t):=\max \{k \in$ $\left.\left.\mathbb{N}\right|_{s_{k+\tau_{k}}}\right\}$ denotes the index of the most recent control input that is available at time $t$, also indicates the possibility of rejecting the packets with large delay. Since the time variable $k$ can be defined from a strictly increased sequence i.e., the queue buffer uses counters to assign the control signals at each node to regulate the flow of packets from the output packet queues to the outgoing link [17].

Under the assumption $\tau_{k} \leq \bar{d} h, \forall k$ and the maximum number of consecutive packet dropout is $\bar{\delta}$ where, $\overline{\mathrm{d}}$ and $\bar{\delta}$ are positive integer, define $l:=\bar{d}+\bar{\delta}$. The following is the discrete equivalent of system (1) at the sampling instant $s_{k}$, 
which will form the basis of the analysis.

$$
x_{k+1}=\Phi x_{k}+\Gamma_{i 0}^{k} u_{i}+\Gamma_{i 1}^{k} u_{i-1}
$$

where, $\Phi=e^{A h}$,

$$
\Gamma_{i 0}^{k}=\sum_{i=0}^{l} \int_{0}^{h-t_{i}^{k}} e^{A s} d s B \text { and } \Gamma_{i 1}^{k}=\sum_{i=0}^{l} \int_{h-t_{i}^{k}}^{h} e^{A s} d s B
$$

for $i \in[k-l, k-l+1, \cdots, k]$

$t_{i}^{k}$ is defined as the instant that the control $u_{i}$ begin to act on the system, and $t_{i}^{k} \in[0, h]$ where, $t_{k-l}^{k}=0$ and $t_{k+1}^{k}=h$. In the following, a switched approach which a combination of that in [8] and [16]. The interval $[0, h]$ will be divided into $p$ equidistant intervals, then $t_{i}^{k} \in[\alpha h / p,(\alpha+1) h / p]$ where $\alpha=0,1, \cdots,(p-1)$. If $p$ is large enough then,

$$
t_{i}^{k}=\beta h / p, \quad \beta=0,1, \cdots, p
$$

With $t_{i}^{k} \leq t_{i+1}^{k}$ and $i \in[k-l, k-l+1, \cdots, k]$.Moreover, $0=t_{k-l}^{k} \leq t_{k-l+1}^{k} \leq \cdots \leq t_{k}^{k} \leq t_{k+1}^{k}=h$ That mean $t_{i}^{k}$ switch in finite set $s$ with $s \in[0, h / l, 2 h / l$, $\cdots,(l-1) h / l, h]$. Hence, (2) contains all possible control inputs that can be activated during the sampling interval $\left[s_{k}, s_{k+1}\right]$, i.e., (2) is sufficient to model the situations in (i) (iii), because each subsystem can be obtained by the definition of $t_{i}^{k}$. Then the control action $u^{*}(t)$ can be described by $u^{*}(t)=u_{i}$ for $t \in\left[s_{k}+t_{i}^{k}, s_{k}+t_{i+1}^{k}\right]$. If the control input dropped or rejected, the latest available control input will be

$$
u^{*}(t)=\left\{\begin{array}{cl}
u_{k-l}, & t \in\left[\mathrm{s}_{\mathrm{k}}, \mathrm{s}_{\mathrm{k}}+\beta_{1} h / p\right] \\
u_{k-l+1}, & t \in\left[\mathrm{s}_{\mathrm{k}}+\beta_{1} h / p, \mathrm{~s}_{\mathrm{k}}+\beta_{2} h / p\right] \\
\vdots \\
u_{k}, \quad t \in\left[\mathrm{s}_{\mathrm{k}}+\beta_{l} h / p,(k+1) h\right]
\end{array}\right.
$$

where, $\beta_{j} \in\{0,1, \cdots, p\}, j=1, \cdots, l$

Since the time delay $\tau_{k}$ is uncertain, $\Gamma_{i 0}^{k}$ and $\Gamma_{i 1}^{k}$ are uncertain matrices. To extract the uncertainties the matrix theory can be applied, see [19].

For $A \in \mathbb{R}^{n \times n}$ we can apply Jordan decomposition on $A$ : $A=T J T^{-1}$ with $J=\left.\operatorname{diag}\left(\lambda_{r}\right)\right|_{r=1} ^{n}$. Here we assume $\lambda_{r}$ are none zero eigenvalues, then the following are obtained

$$
\begin{gathered}
\Gamma_{i 0}^{k}=\Gamma_{0}^{k}+\Delta \Gamma_{i 0}^{k}=\Gamma_{0}^{k}+D \Delta \Gamma_{i}^{k} E \\
\Gamma_{i 1}^{k}=\Gamma_{1}^{k}+\Delta \Gamma_{i 1}^{k}=\Gamma_{1}^{k}+D \Delta \Gamma_{i}^{k} E
\end{gathered}
$$

where,

$$
\begin{aligned}
& \Gamma_{0}^{k}=-\operatorname{Tdiag}\left\{1 / \lambda_{1}, \cdots, 1 / \lambda_{r}\right\} E, \quad E=T^{-1} B \\
& \Gamma_{1}^{k}=\operatorname{Tdiag}\left\{e^{\lambda_{1} h} / \lambda_{1}, \cdots, e^{\lambda_{r} h} / \lambda_{r}\right\} E, \\
& D=\operatorname{Tdiag}\left\{e^{\lambda_{1} \alpha_{1}} / \lambda_{1}, \cdots, e^{\lambda r \alpha_{r}} / \lambda_{r}\right\} E, \\
& \Delta \Gamma_{i}^{k}=\operatorname{diag}\left\{e^{\lambda_{1}\left(h-t_{i}^{k}-\alpha_{1}\right)}, \cdots, e^{\lambda_{r}\left(h-t_{i}^{k}-\alpha_{r}\right)}\right\}
\end{aligned}
$$

Let,

$$
\begin{gathered}
D_{1}=[D-D], E_{1}=\operatorname{diag}(E, E) \text { and } \\
\Delta \Gamma^{\mathrm{k}}=\operatorname{diag}\left(\Delta \Gamma_{i}^{k}, \Delta \Gamma_{i}^{k}\right)
\end{gathered}
$$

Then,

$$
\left[\Delta \Gamma_{0 i}^{k} \quad \Delta \Gamma_{1 i}^{k}\right]=D_{1} \Delta \Gamma^{k} E_{1}
$$

Now rewrite model (2) in the state space form with state vector $\zeta_{k}=\left[x_{k}, u_{k-1}, u_{k-2}, \cdots, u_{k-l}\right]$ then,

$$
\begin{aligned}
\zeta_{k+1} & =\tilde{A} \zeta_{k}+\tilde{B} u_{k} \\
y_{k} & =\tilde{C} \zeta_{k}
\end{aligned}
$$

where,

$$
\begin{aligned}
\tilde{A} & =\left[\begin{array}{ccccc}
\Phi & \Gamma_{1}^{k} & \Gamma_{2}^{k} & \cdots & \Gamma_{l}^{k} \\
0 & 0 & 0 & \cdots & 0 \\
0 & I & 0 & \cdots & 0 \\
\vdots & & \ddots & & \vdots \\
0 & 0 & & I & 0
\end{array}\right], \quad \tilde{B}=\left[\begin{array}{c}
\Gamma_{0}^{k} \\
I \\
0 \\
\vdots \\
0
\end{array}\right] \\
\tilde{C} & =\left[\begin{array}{lllll}
C & 0 & 0 & \cdots & 0
\end{array}\right], \quad \Gamma_{i}^{k}=\Gamma_{i 0}^{k}+\Gamma_{(i-1) 1}^{k}
\end{aligned}
$$

Remark 2: More precise bound on $\Delta \Gamma^{k}$ can be achieved based on the bound on $\alpha_{r}$ i.e., the selection of $\alpha_{r}$ can make $\lambda_{r}\left(h-t_{i}^{k}-\alpha_{r}\right) \leq 0$. In particular any value $\delta$ can be used to limit the term $\sigma_{\max }\left(\Delta \Gamma^{k}\right)$. In [20] shown that the uncertainty $\Delta \Gamma^{k}$ is norm bounded on a compact $\delta \tau_{k} \epsilon\left[\delta \tau_{\text {min }} ; \delta \tau_{\max }\right]$, thus there exist a scalar $\gamma$ such that,

$$
\sup _{\tau_{\max } \in[0, \bar{d} h]} \sigma_{\max }\left(\Delta \Gamma^{k}\right) \leq \gamma \Leftrightarrow\left(\Delta \Gamma^{k}\right)^{T} \Delta \Gamma^{k} \leq \gamma^{2} I
$$

For non-diagonal $J$, similar procedure can be developed to take the advantage of using the property of matrix function structure [8].

For this work we consider a state feedback controller of the form

$$
u_{k}=-K x_{k}=\left[\begin{array}{llll}
-K & 0 & \cdots & 0
\end{array}\right] \zeta_{k}=-\tilde{K} \zeta_{k}
$$

Applying (8) to the plant (6), result in the following closed loop system.

$$
\begin{aligned}
\zeta_{k+1} & =(\tilde{A}-\tilde{B} \tilde{K}) \zeta_{k}+\Delta \tilde{A}_{c l}\left(t_{i}^{k}, \tilde{K}\right) \zeta_{k} \\
& =\left[\tilde{A}_{c l}\left(t_{i}^{k}\right)+\Delta \tilde{A}_{c l}\left(t_{i}^{k}\right)\right] \zeta_{k}
\end{aligned}
$$

where,

$$
\begin{aligned}
\tilde{A}_{c l}\left(t_{i}^{k}\right) & =\left[\begin{array}{ccccc}
\Phi-\Gamma_{0}^{k} K & \Gamma_{1}^{k} & \Gamma_{2}^{k} & \cdots & \Gamma_{l}^{k} \\
-K & 0 & 0 & \cdots & 0 \\
0 & I & 0 & \cdots & 0 \\
\vdots & & \ddots & & \vdots \\
0 & 0 & & I & 0
\end{array}\right] \\
\Delta \tilde{A}_{c l}\left(t_{i}^{k}\right) & =\left[\begin{array}{cc}
\Delta \bar{\Gamma}_{i 0}^{k} K & \Delta \bar{\Gamma}_{i 1}^{k} \\
0_{l \times n} & 0_{l \times l}
\end{array}\right]=\left[\begin{array}{c}
D_{1} \\
0
\end{array}\right] \Delta \Gamma^{k} Q
\end{aligned}
$$

From (10) the following derivation is true

$$
\begin{aligned}
& \tilde{A}_{c l}\left(t_{i}^{k}\right)=\tilde{A}-\tilde{B} \tilde{K}, \\
& \Delta \tilde{A}_{c l}\left(t_{i}^{k}\right)=\Delta \tilde{A}-\Delta \tilde{B} \tilde{K}=X_{1} \Delta \Gamma^{k} Q_{1} \\
& \text { with } Q_{1}=-E_{1}\left[\tilde{K}+X_{2}\right] \text { where, } \\
& X_{1}=\left[\begin{array}{c}
D_{1} \\
0
\end{array}\right] \text { and } X_{2}=\left[\begin{array}{ll}
0 & I
\end{array}\right]
\end{aligned}
$$


In the next section, a criterion is given to guarantee the robust stability of closed loop system (9), and controller design.

\section{MAIN RESULTS}

In this section, we will present a sufficient condition for the robust stability of system (9). For this purpose, we will introduce the following lemma.

Lemma1. [21] Let $A, E, F$ and $H$ is real matrices of appropriate dimensions with $\|F\|_{2} \leq 1$. Then for $P>0$ and scalar $\varepsilon>0$ satisfying $\varepsilon I_{n}-E^{T} P E>$, we have

$$
\begin{aligned}
& (A+E F H)^{T} P(A+E F H) \\
& \leq A^{T} P A+A^{T} P E\left(\varepsilon I_{n}-E^{T} P E\right)^{-1} E^{T} P+\varepsilon H^{T} H
\end{aligned}
$$

Theorem 1. If there exist asymmetric positive definite matrix $P \in R^{n \times n}$ and positive scalar $\varepsilon>0$ such that the following inequality

$$
\left[\begin{array}{cc}
A_{c l}^{T} P A_{c l}-P+\varepsilon\left(\gamma^{2}\right) Q_{1}^{T} Q_{1} & A_{c l}^{T} P X_{1} \\
* & -\left(\varepsilon I_{n}-X_{1}^{T} P X_{1}\right)
\end{array}\right]<0
$$

holds, the controller (8) robustly stabilize the NCS (6) for arbitrary time varying delays and uncertainties

Proof: in order to find the sufficient condition for the stability of the system with arbitrary time varying delay $\tau_{k} \in[0, \bar{d} h]$, therefore a system with finite number of LMIs can be solved Define a quadratic Lyapunov function $V\left(\zeta_{k}\right)=\zeta_{k}^{T} P \zeta_{k}$, differentiate along the trajectory of the closed loop system (9) will obtain the negative definite matrix

$$
Q=\left(\tilde{A}_{c l}\left(t_{i}^{k}\right)+\Delta \tilde{A}_{c l}\left(t_{i}^{k}\right)\right)^{T} P\left(\left(\tilde{A}_{c l}\left(t_{i}^{k}\right)+\Delta \tilde{A}_{c l}\left(t_{i}^{k}\right)\right)-P\right.
$$

To take advantage of lemmalwe define,

$$
S \triangleq\left[\varepsilon I_{n}-X_{1}^{T} P X_{1}\right]^{-(1 / 2)} X \tilde{A}_{c l}-\left[\varepsilon I_{n}-X_{1}^{T} P X_{1}\right]^{(1 / 2)} \Delta \Gamma^{k} Q_{1}
$$

then,

$$
\begin{aligned}
0 \leq & S^{T} S \\
= & \left(\tilde{A}_{c l}\right)^{T} P X_{1}\left[\varepsilon I_{n}-X_{1}^{T} P X_{1}\right]^{-(1 / 2)} X_{1} P \tilde{A}_{c l} \\
& +Q_{1}^{T} \Delta \Gamma^{T}\left[\varepsilon I_{n}-X_{1}^{T} P X_{1}\right] \Delta \Gamma Q_{1} \\
& -\left\{\left(\tilde{A}_{c l}\right)^{T} P X_{1} \Delta \Gamma Q_{1}+Q_{1}^{T} \Delta \Gamma^{T} X_{1}^{T} P \tilde{A}_{c l}\right\}
\end{aligned}
$$

Adding the term $\left(\tilde{A}_{c l}\right)^{T} P \tilde{A}_{c l}+\left[Q_{1}^{T}\left(\Delta \Gamma^{k}\right)^{T} X_{1}^{T}\right] P\left[X_{1} \Delta \Gamma^{k} Q_{1}\right]$ to the both side in order to complete the square, then to form $Q$ in left side add $(-P)$ to the both side, result in

$$
\begin{aligned}
Q= & \left(\tilde{A}_{c l}+X_{1} \Delta \Gamma^{k} Q_{1}\right)^{T} P\left(\tilde{A}_{c l}+X_{1} \Delta \Gamma^{k} Q_{1}\right)-P \\
\leq & \left(\tilde{A}_{c l}\right)^{T} P \tilde{A}_{c l}+\left(\tilde{A}_{c l}\right)^{T} P X_{1}\left[\varepsilon I_{n}-X_{1}^{T} P X_{1}\right]^{-1} X_{1}^{T} P \tilde{A}_{c l} \\
& -\varepsilon Q_{1}^{T}\left(\Delta \Gamma^{k}\right)^{T} \Delta \Gamma^{k} Q_{1}
\end{aligned}
$$

From (7) we have $\left(\Delta \Gamma^{k}\right)^{T} \Delta \Gamma^{k}<\gamma^{2} I_{n}, \forall \tau_{k} \in[0, \bar{d} h]$, inequality (12) become, become, become, become,

$$
\begin{aligned}
Q \leq & \left(\tilde{A}_{c l}\right)^{T} P \tilde{A}_{c l}-P+\varepsilon \gamma^{2} Q_{1}^{T} Q_{1} \\
& +\left(\tilde{A}_{c l}\right)^{T} P X_{1}\left[\varepsilon I_{n}-X_{1}^{T} P X_{1}\right]^{-1} X_{1}^{T} P \tilde{A}_{c l}
\end{aligned}
$$

Since the robust stability condition will be satisfied if and only if the RHS of (13) is negative definite, then the closed loop system is asymptotically stable $\forall \tau_{k} \in\left[0, \tau_{\text {max }}\right]$. Then according to Schur complement lemma [1] allows readily obtain inequality (11)

Remark 3: Based on the conditions of stability in Theorem1, the conditions of existence for the state feedback controllers are presented in the following theorem.

Theorem 2: Given a scalar $\gamma$, there exist a state feedback controller such that the closed loop system (9) is robust asymptotically stable with bound $\gamma$, if there exist matrices $Y>0, \widetilde{R}$, and constant $\lambda>0$, satisfying

$$
\left[\begin{array}{cccc}
-Y & \left(N_{0} \tilde{R}+X_{2} Y\right)^{T} & (\tilde{A} Y-\tilde{B} \tilde{R})^{T} & 0 \\
(\tilde{A} Y-\tilde{B} \tilde{R}) & -\lambda I & 0 & 0 \\
\left(N_{0} \tilde{R}+X_{2} Y\right) & 0 & -Y & \lambda X_{1}^{T} \\
0 & 0 & \lambda X_{1} & -\lambda I
\end{array}\right]<0
$$

Then the feedback gains can be obtained by:

$$
\widetilde{K}=\tilde{R} Y^{-1}
$$

Proof: From (9) we have $\widetilde{K}=\left[\begin{array}{lll}-K & \cdots & 0\end{array}\right], \widetilde{K} \in$ $R^{m \times(n+l)}$ then, the parameter $\Delta \tilde{A}_{c l}\left(t_{i}^{k}\right)$ can be rewritten as,

$$
\begin{aligned}
\tilde{A}_{c l}\left(\Delta \tau_{i}\right) & =\Delta \tilde{A}-\Delta \tilde{B} \widetilde{K}=X_{1} \Delta \Gamma^{k} Q_{1} \\
\text { with } Q_{1} & =\left[N_{0} \tilde{K}+X_{2}\right] \text { where, } \\
X_{1} & =\left[\begin{array}{c}
M_{0} \\
0
\end{array}\right] \text { and } X_{2}=\left[\begin{array}{ll}
0 & N_{0}
\end{array}\right]
\end{aligned}
$$

Then from (11) the closed loop system is robustly stable if

$$
\left[\begin{array}{cc}
(\tilde{A}-\tilde{B} \tilde{K})^{T} P(\tilde{A}-\tilde{B} \tilde{K})-P & (\tilde{A}-\tilde{B} \tilde{K})^{T} P X_{1} \\
+\varepsilon\left(\gamma^{2}\right)\left(N_{0} \tilde{K}+X_{2}\right)^{T}\left(N_{0} \tilde{K}+X_{2}\right) & \\
* & -\left(\varepsilon I_{n}-X_{1}^{T} P X_{1}\right)
\end{array}\right]<0
$$

Define $Y=P^{-1}$, and Pre- and post-multiplying the last inequality by $\operatorname{diag}(Y, I)$ yields,

$$
\left[\begin{array}{cc}
(\tilde{A} Y-\tilde{B} \tilde{R})^{T} P(\tilde{A} Y-\tilde{B} \tilde{R})-P & (\tilde{A} Y-\tilde{B} \tilde{R})^{T} P X_{1} \\
+\varepsilon\left(\gamma^{2}\right)\left(N_{0} \tilde{R}+X_{2} Y\right)^{T}\left(N_{0} \tilde{R}+X_{2} Y\right) & \\
* & -\left(\varepsilon I_{n}-X_{1}^{T} P X_{1}\right)
\end{array}\right]<0
$$

Then, we can obtain the following structure for $K$

$$
\tilde{R}=\tilde{K} Y=\left[\begin{array}{llll}
-K & 0 & \cdots & 0
\end{array}\right]\left[Y_{i j}\right] \text { with } i j \in(1,2, \ldots, n+l)
$$

Let $\lambda=\frac{1}{\varepsilon\left(\gamma^{2}\right)}>0$ and applying shur complement yield inequality (14) this end the proof.

Remark 4. It can be seen from (7) that the scalar $\gamma$ require significant computational, we can be obtained numerically an upper bound of the maximal singular value of $\Delta \Gamma^{k}$, by computing offline the singular value of $\Delta \Gamma^{k}$ over tight grid verifying $\tau_{k}=[0 ; \bar{d} h]$. A detailed computational method for $\gamma$ is given in [19]. Henceforth, rather than using $\gamma$, any other $\gamma_{1} \geq \gamma$ can be used in order to find more conservative 
allowable delay. Adopting approach similar to [8], the feasibility of (11) can be used to compute the maximum delay $\tau_{\max }$ that can be tolerated for the given feedback gain; hence we have the following optimization problem.

$$
\tau=\max \left(\tau_{\max }\right)
$$

Subject to $\gamma=\sup _{\tau_{\max } \in[0, \bar{d} h]} \sigma_{\max }\left(\Delta \Gamma^{k}\right), \forall \tau_{k} \in\left[0, \tau_{\max }\right)$ and (11)

Remark 5. Recently, much attention has been focused on analysis and synthesis of networked control systems NCSs, and many stability criteria can be traced in [22], [23]. In [24], an NCS with limited bandwidths was modeled as a switched system with time-varying delay, and a similar switching law had been used to stabilize the NCS, which shows that the result given in this paper may also be applicable to such system.

\section{NUMERICAL EXAMPLES}

To illustrate the proposed theoretical results, a numerical example is considered in this section. The system setup is given by Fig. 1. The continuous-time model:

$$
\dot{x}(t)=\left[\begin{array}{cc}
0 & 1 \\
-3 & -4
\end{array}\right] x(t)+\left[\begin{array}{l}
0 \\
1
\end{array}\right] u(t)
$$

The sampling period is chosen as $h=0.05 s$. Assume the delay $\tau_{k} \leq 2 h$ that is $\bar{d}=2$ when no packet dropout $l=2$, then the actuator receives at most 3 control input during $\left[s_{k}, s_{k+1}\right]$. Clearly from (2) the instant of control inputs $u_{k}, u_{k-1}$ can be denoted as $t_{0}^{k}$ and $t_{1}^{k}$, respectively. Suppose that $\alpha_{r}$ uniformly distributed at the interval $[-h / 2, h / 2] \quad$ then, $\left(\Delta \Gamma^{k}\right)^{T} \Delta \Gamma^{k} \leq \gamma^{2} I \quad$ with $\quad \gamma \in$ $[0.9743,1.0747]$, the initial state as $x_{0}=\left[\begin{array}{ll}1 & -0.5\end{array}\right]^{T}$, then one obtains

$$
\begin{gathered}
\Phi=\left[\begin{array}{cc}
1.0000 & 1.0513 \\
0.8607 & 0.8187
\end{array}\right], \Gamma_{0}^{\mathrm{k}}=\left[\begin{array}{l}
0.3333 \\
0.0000
\end{array}\right] \\
E=\left[\begin{array}{ll}
0.7071 \\
1.5811
\end{array}\right] \Gamma_{1}^{k}=\left[\begin{array}{c}
-0.3322 \\
0.0453
\end{array}\right] \\
\mathrm{D}=\left[\begin{array}{cc}
-0.6896 & 0.0978 \\
0.6896 & -0.2934
\end{array}\right]
\end{gathered}
$$

and

$$
\Delta \Gamma_{i}^{k}=\Delta \Gamma_{i}^{k}=\operatorname{diag}\left\{e^{-\left(0.05-t_{i}^{k}-\alpha_{1}\right)}, \cdots, e^{-3\left(0.05-t_{i}^{k}-\alpha_{r}\right)}\right\}
$$

First, we analyze the feasibility of the LMI in (11) for various values of $\tau_{k}$ and $\gamma$. The delay is considered to be time varying and the LMI is solved for $\tau_{\max } \in[0,0.8 h]$, in this case no packet rejection will occur since $\tau_{\max }<h$. Here we remark the feasibility of (11) is sensitive to the value of $\gamma$ for the nominal gain $\left[\begin{array}{ll}1.5 & 2\end{array}\right]$.

For the case of $\tau_{\max } \in[0,2.6 h]$ or when $\bar{\delta}=1$ and $\tau_{\text {max }}=1.6 h$, or when $\bar{\delta}=2$ and $\tau_{\text {max }}=0.6 h$, this is true as long as $l:=\bar{d}+\bar{\delta}$. In this case where large delay or packet drop out, or both together occurs, the stabilizing controller can be found for $\gamma=0.9743$ then,

$$
\begin{aligned}
Y & =\left[\begin{array}{lll}
2.0800 & 12.0560 & 7.0640 \\
5.0700 & 2.3600 & 16.0620 \\
0.1940 & 8.0500 & 5.0020
\end{array}\right] \\
R & =\left[\begin{array}{lll}
12.0500 & 1.0046 & 11.0600
\end{array}\right]
\end{aligned}
$$

Then the controller is calculated as,

$$
K=\left[\begin{array}{ll}
-2.3652 & 1.0214
\end{array}\right]
$$

\section{CONCLUSION}

The proposed method is to deal with the problem of congestion arising in NCSs. A simple scheme is used in order to eliminate the effect of the congestion, a deterministic packet drops out is considered. Combined delay switching and parameter uncertainty based method is proposed, and then sufficient conditions on the stability and stabilization have been derived in terms of LMIs. Stabilizing feedback controller can be constructed via the feasible solution of a set of LMIs. Moreover, for NCSs with data packet dropout and delays, sufficient conditions on stabilization of the NCSs have been established in a similar manner. The results obtained in this paper suggest that one may drop data packet at a certain rate to save network bandwidth while preserving the stability of the NCS.

\section{REFERENCES}

[1] J. P. Hespanha, P. Naghshtabrizi, and Y. G. Xu, "A survey of recent result in networked control system," Proceedings of the IEEE, vol. 95, no. 1, pp. 138-162, 2007.

[2] E. Fridman and U. Shaked, "Delay-dependent stability and $\mathrm{H}_{\mathrm{inf}}$ control: Constant and time-varying delays," International Journal of Control, vol. 76, no. 1, pp. 48-60, 2003.

[3] M. B. G. Cloosterman, N. van de Wouw, W. P. M. H. Heemels, and H. Nijmeijer, "Stability of networked control systems with uncertain time-varying delays," IEEE Transaction on Automatic Control, vol. 54, no. 7, pp. 1575-1580, 2009.

[4] J. Y. Yu, L. Wang, G. F. Zhang, and M. Yu, "Output feedback stabilization of networked control system via switched system approach," International Journal of Control, vol. 82, no. 9, pp. 1665-1677, 2009.

[5] L. Dritsas and A. Tzes, "Robust stability analysis of networked systems with varying delays," International Journal of Control, vol. 82, no. 12, pp. 2347-2355, December 2009.

[6] Y. S. Moon, P. Park, W. H. Kwon, and Y. S. Lee, "Delay-Dependent robust stabilization of uncertain state delayed systems," International Journal of Control, vol. 74, no. 14, pp. 1447-1455, 2001.

[7] G. Walsh, H. Ye, and L. Bushnell, "Stability analysis of network control systems," IEEE Transactions on Control Systems Technology, vol. 10, no. 3, pp. 438-446, 2002.

[8] M. B. G. Cloosterman, L. Hetel, N. van de Wouw, W. P. M. H. Heemels, J. Daafouz, and H. Nijmeijer, "Controller synthesis for networked control systems," Automatica, vol. 46, pp. 1584-1594, 2010 .

[9] S. S. Hu and Q. X. Zhu, "Stochastic optimal control and analysis of stability of networked control systems with long delay," Automatica, vol. 39, pp. 1877-1884, 2003.

[10] Y. L. Wang and G. H. Yang, "State feedback control synthesis for networked control systems with packet dropout," Asian Journal of Control, vol. 11, pp. 49-58, 2009.

[11] J. N. Li, Q. L. Zhang, and M. Cai, "Modelling and robust stability of networked control systems with packet reordering and long delay," International Journal of Control, vol. 82, iss. 10, pp. 1773-1785, 2009.

[12] R. Luck and A. Ray, "An observer-based compensator for distributed delays,” Automatica, vol. 26, pp. 903-908, 1990.

[13] J. Baillieul and P. J. Antsaklis, "Control and communication challenges in networked real-time systems," Proceedings of the IEEE, vol. 95, no. 1, pp. 9-28, 2007. 
[14] R. Rajesh, M. Lakshmanan, and V. N. Mohammed, "Implementation of networked control systems using TCP/IP," International Journal of Computer Applications, vol. 18, no. 2, pp. 1-5, 2011.

[15] E. Dedu, G. Bise, and J. Bourgeois, "An analysis of congestion controls in centralized control systems," in Proc. Network Games, Control and Optimization (NetGCooP), 6th International Conference, 2012, pp. $121-126$.

[16] Y. L. Wang and G. H. Yang, " $H_{\text {inf }}$ controller of state feedback networked control systems with long delay and packet dropout," in Proc. IEEE International Conference on Control Applications, CCA Singapore, 2007, pp. 65-70.

[17] Yang and Y. J. Wang, "Modeling and control for NCS with timevarying long delays," in Proc. the 4th International Conference on Machine Learning and Cybernetics, Guangzhou, August 2005, pp. 1407-1411.

[18] J. Xiong and J. Lam, "Stabilization of linear systems over networks with bounded packet loss," Automatica, vol. 43, no. 1, pp. 80-87, 2007.

[19] S. Li, Z. Wang, and Y. Sun, "Observer-based compensator design for networked control systemswith long delays," in Proc. 30th Conference of the IEEE Industrial Electronics Society, Busan, Korea, 2004, pp. 678-683.

[20] L. Hetel, J. Daafouz, J. P. Richard, and M. Jungers, "Delay dependant sampled-data control based on delay estimates," Systems \& Control Letters, vol. 60, pp. 146-150, 2011.

[21] Y. Wang, Q. S. Zeng, and C. S. Fu, "Modeling and control of networked control systems with random delays," in Proc. HSCC Conf., Berlin, 2005, pp. 655-666.

[22] M. C. F. Donkers, W. P. M. H. Heemels, N. van de Wouw, and L. Hetel, "Stability analysis of networked control systems using a switched linear systems approach," IEEE Trans. Autom. Control, vol. 56, no. 9, pp. 2101-2115, 2011.

[23] Y. L. Wang and Q. L. Han, "Quantitative analysis and synthesis for networked control systems with non-uniformly distributed packet dropouts and interval time-varying sampling periods," Int. J. Robust.
Nonlinear Control, 2013.

[24] M. Souza, G. S. Deaecto, J. C. Geromel, and J. Daafouz, "Self-triggered linear quadratic networked control," in Proc. Preprints of the 20th Mediterranean Conf. on Contr. \& Automation, Barcelona, 2012, pp. 942-947.

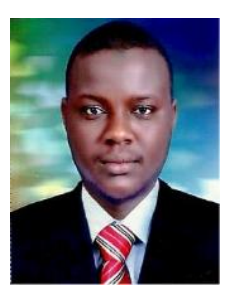

Yasir B. Hamdan received the B.Sc. Eng. degree in electronic engineering Tech. from the University of Gezira, Madani in 2003 and the M.Sc. degree in microprocessor and control system from the Sudan University of Science and Technology, Khartoum in 2007. Mr. Hamdan is pursuing doctorate in philosophy in control engineering and control theory from the University of Science and Technology of China, Hefei. And he is working as a research fellow. He worked as a lecturer at the Electrical Engineering Department, RedSea University, PortSudan. He had published a research article in on-line journal 2013, he communicates another research article in international control journal's, he already published a part of his research in international conferences. His thrust research area is control systems design and robust control, networking control system, time delay systems and congestion control.

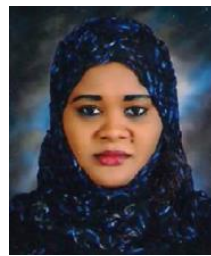

Samah Omer Altayeb received the B.Sc. Eng. degree in electronic engineering Tech. from the University of Gezira, Madani in 2007. She is currently pursuing a master degree in automation at the University of Science and Technology of China, with the interest in the biomedical engineering studies. She has presented several works at the national and international level on the biomedical aspects. 